Diánoia, vol. 28, no. 28, 1982

\title{
PRESENCIA DEL LÍMITE. WITTGENSTEIN Y "LO MÍSTICO" ${ }^{1}$
}

RaMón Xirau

\author{
INSTITUTO DE INVESTIGACIONES FILOSOFICAS \\ Universidad Nagtonal autónoma de MÉxico
}

"Lo inefable (aquello que me parece misterioso y que no me atrevo a expresar) proporciona quizá el transfondo sobre el cual adquiere significado lo que yo pudiera expresar." Ludwig Wittgenstein, Observaciones (1931).2

La querella es antigua pero no deja de ser absolutamente actual. Con el cristianismo se vuelve crucial todo lo que toca a las relaciones, o ausencia de ellas, entre la fe y la razón. ${ }^{3}$ Aquellos que temen que la razón humana pueda destruir la $\mathrm{fe}$, o aquellos que piensan que la fe nunca podrá conciliarse con la razón, niegan la posibilidad del pensamiento racional en materias religiosas y afirman que el único conocimiento auténtico es el que proporciona la fides, es decir la fe doblada de confianza. Entre los siglos II y III, Tertuliano inauguró el fideísmo para que el fideísmo reapareciera a lo largo de la Edad Media y viviera hasta los tiempos modernos y contemporáneos en dos pensadores tan hondos como extremosos: Kierkegaard, Unamuno. Kierkegaard, el "caballero de la fe", usaba la razón para mostrar la incapacidad de esta misma razón y decía -cuestión de vida o muerte_ que el verdadero hombre, el hombre reli-

1 Me ocupa aquí el primer Wittgenstein, el del Tractatus y de los Notebooks, escritos en los mismos años. Sobre lo "místico" en Wittgenstein hemos escrito en México Luis Villoro y yo - mi primer escrito sobre el tema figura en el libro Palabra y silencio (Siglo XXI, 1968 y 1972). Hoy no me satisface del todo aquel texto. De ahi estas nuevas refiexiones. En 1975 Villoro escribió un texto de primera sobre el tema ("Lo indecible en el Tractatus", revista Critica, Universidad Nacional Autónoma de México, vol. VII, No 19). En 1968 me ocupé de discernir entre lo "decible" y lo "indecible" en el Tractatus. Ahora apenas me refiero a lo "decible" por dos motivos: quiero entrar de lleno en el problema de "lo místico"; por lo que toca a lo "decible" remito al texto de Villoro, mucho más detallado que el mío de 1968 y también mucho mejor. En cuanto a lo "indecible", dos diferencias entre Villoro y yo: Villoro, de manera intencional, no toma en cuenta los Notebooks; para mi resultan fundamentales; Villoro parece pensar que lo "místico" no puede estar fuera del mundo; por mi parte creo que, en varios momentos, Wittgenstein parece pensar que lo "místico" está precisamente fuera del mundo. Sea como sea, para entender lo "místico" es indispensable recordar la presencia del "límite" en el Tractatus. En efecto, Wittgenstein no piensa, como lo pensó Russell, que existan metalenguajes y metalengluajes de metalenguajes, etcétera. En el Tractatus lo que importa es el lenguaje y el límite del lenguaje.

2 L. Wittgenstein, Observaciones, recopilado por G. H. von Wright. Traducción de Elsa Cecilia Frost, Siglo XXI, México, 1981.

3 Naturalmente, la querella se encuentra en el mundo musulmán y en la tradición del pensamiento judio. 
gioso, era el que podía realizar el saltum mortale, el salto de lo finito a lo infinito, cosa de fe, confianza, amor. Unamuno, en su lucha a más que brazo partido, encontraba consuelo en las palabras evangélicas: "Señor, creo; ayuda a mi incredulidad." 4

Ante el fideísmo algunos pensaron que podía racionalizarse totalmente la fe: así, en el siglo xil y en el mundo musulmán, Averroes y, como nadie antes que él, Hegel, quien, por cierto, negaba el valor de las metáforas en cuestiones filosóficas y se veía obligado a usarlas cuando más creía haber alcanzado la razón, en las vías hacia el espíritu absoluto y, definitivamente, en el espiritu absoluto. ${ }^{\circ}$

Dos extremos: afirmar únicamente la fe; afirmar únicamente la razón. Recordemos, sin embargo, que la actitud más frecuente durante la Edad Media fue la que intentó conciliar la fe y la razón. Para el creyente la fe será siempre superior a la razón, pero ésta podrá venir en ayuda de la fe. Tal actitud parte de San Agustín cuando dice, ad hominem: "Credo ut intelligas; intellige ut credas." $\mathrm{Y}$, por distintos que sean entre sí, los filósofos medievales, agustinianos o no, tenderán a conciliar fe, razón y amor: San Anselmo, Santo Tomás, San Buenaventura, Ramón Lull, Duns Escoto entre ellos. ${ }^{8}$

Si tratamos de ver atentamente el desarrollo de la filosofía occidental a partir del Renacimiento, resultará claro que la querella fe-razón no es una antigualla ni una reliquia. Descartes no olvidaba la fe pero la dejaba en manos de los teólogos, acaso con temor, para ocuparse en fundar una filosofía de pretensiones puramente racionales. Descartes creia, pero también pensaba que su creencia no debía inmiscuirse con sus argumentos claros, distintos (es decir, evidentes) y lógicos. La actitud cartesiana no es la de quien niega la fe sino la de quien escinde y separa razón y fe, actitud que, en Descartes, acaso no sea reductivista, pero que podrá llegar a serlo en sus semi-seguidores: tal el caso de La Mettrie. Pero acaso quien más claramente y apasionadamente expresa la actitud de los modernos sea Kant, este Kant mucho más lleno de emociones de lo que podría parecer a primera vista.

En la primera de sus críticas, Kant intenta encontrar fundamentos universales y necesarios para el conocimiento. Pero, como sabemos gra-

4 Carlos Blanco Aguinaga mostró en El Unamuno contemplativo que existe un Unamuno lleno de paz o, por lo menos, deseoso de paz. Es cierto. No lo es menos que este Unamuno que contempla lucha con el Unamuno que medita de tal manera que a la agonía que entraña el conflicto entre la razón y el corazón hay que añadir la nueva lucha entre la "paz" y la "guerra".

5 Cf. mi Entre ídolos y dioses, El Colegio Nacional de México, 1980.

6 Las primeras búsquedas de un apoyo racional para la fe deben buscarse en Orígenes -patrística gxiega- y en Justino mártir - patrística latina. Ambos encuentran la razón que buscan en la filosofía griega. 
cias a la última parte de la Crítica de la razón pura y gracias a la segunda crítica, la de la razón práctica, lo fundamental y, yo diría, vital, es para Kant limitar el alcance del conocimiento para abrir de par en par las ventanas de la moral y de una metafísica que, por "postulada" que sea, no deja de ser metafísica. Dos preguntas son vitales: "¿qué debo hacer?", ¿qué puedo esperar?". Es decir, a Kant, al que Unamuno llamaba el hombre Kant, al Kant de acentos pietistas le importaban las normas para la moral y le importaba la inmortalidad del alma, y le importaba la existencia de Dios. Ciertamente, Kant trató de ver "la religión dentro de los límites de la mera razón" y buscó una "fe racional", pero esta fe, esta fe racionalizada nacía de experiencias vitales, de un verdadero acto de fe que no deja de ser una suerte de saltum mortale. ${ }^{7}$

Pero, ¿qué tienen que ver todas estas actitudes y tradiciones con el pensamiento de Wittgenstein? A primera vista, nada; en verdad, todo.

Wittgenstein parece haber sido hombre de poca fe, lo cual no significa que esta fe poca no le angustiara y acongojara. Así, en 1928, Wittgenstein escribía a su amigo Engelmann: "claro todo se reduce a que no tengo fe". De hecho, el Tractatus ha sido repetidamente mal interpretado y esto es especialmente cierto cuando vemos que los filósofos anglosajones -y ahora sus discípulos españoles, mexicanos, argentinos- se preocupan más por los problemas lógicos y epistemológicos que planteaba (o resolvía) el Tractatus que por lo que ocupaba y preocupaba vitalmente a Wittgenstein. La verdad es que no se ha prestado suficiente atención a lo que Wittgenstein escribe. Ya en el prólogo al Tractatus escribía: "Opino que los problemas han sido, en lo esencial, finalmente resueltos", pero añadía -y la añadidura es crucial: "Si no estoy equivocado en esto, el valor de este trabajo consiste, en segundo lugar, en el hecho de que muestra cuán poco se ha hecho cuando se han resuelto los problemas." 8 Pues bien, lo que a Wittgenstein le interesaba de verdad era precisamente esto que no habia podido hacer; le interesaba, en efecto, el mundo de lo que se muestra pero que no se demuestra, el mundo de lo "místico", de lo "indecible": la presencia, seguramente nacida de una experiencia, de los límites, del límite.

7 Véase especialmente: Critica de la razón pura, Prefacio a la segunda edición y, sobre todo, Doctrina trascendental del método, capítulo II, "Canon de la razón pura". partes II y III.

8 El subrayado es mío. 
Bertrand Russell se dio muy pronto cuenta de las tendencias místicas de Wittgenstein. No dejaron de alarmarle. En la introducción al Tractatus, introducción que no fue nada del gusto de Wittgenstein, hacía notar Russell que Wittgenstein se las ingeniaba para decir mucho acerca de lo que no se puede decir, y en una carta a Lady Ottoline escribía: "Habja observado en su libro un sabor a misticismo, pero me ha sorprendido que se haya convertido en un místico completo." ${ }^{9} \mathrm{Y}$ añadía: "Creo que lo que más le gusta en la mística es el poder que ésta tiene de permitirle dejar de pensar." En buena medida Wittgenstein habla contestado a Russell cuando escribía en una carta: "Temo que (Russell) no haya captado mi argumento principal, del cual todo el asunto de las proposiciones lógicas no es sino un corolario. El punto principal es la teoría acerca de lo que puede expresarse (Gesagt) mediante proposiciones, es decir, mediante el lenguaje (o, lo que es lo mismo, aquello que puede ser pensado y lo que no puede ser pensado)" a diferencia de "lo que no puede expresarse sino que solamente puede ser mostrado (gezeigt); lo cual es, para mí el asunto cardinal de la filosofía". ${ }^{10}$

A lo que se dice y puede decirse (proposiciones significativas de las ciencias naturales, pseudo-proposiciones de las matemáticas y aun proposiciones de carácter ontológico) se contrapone lo que se muestra y esto es "lo místico". Lo místico es lo que está en el límite o más allá del límite. Para entender el misticismo de Wittgenstein, que nada tiene que ver con las místicas de los grandes creyentes - Bernardo, Eckhart, Rysbrock, Suso o San Juan de la Cruz- es indispensable precisar la noción de límites, y aclarar así, hasta donde se pueda, el sentido de lo mostrable no demostrable. Antes de hacerlo es necesario dar un rodeo para mejor situar el pensamiento de Wittgenstein y sobre todo de aquella Viena de Wittgenstein que analizaron Janik y Toulmin.

Vayan, así, dos recordatorios: los que remiten a la Viena de Wittgenstein y los que remiten a la vida del filósofo en su ciudad de Viena. ${ }^{11}$ Ya desde 1917 es visible el derrumbe del imperio austrohúngaro. Esta caída y derrumbe ponen en crisis a toda una cultura y hacen que sus mejores representantes asuman una actitud precisamente crítica.

Escritores, músicos, arquitectos, pintores, tienden a exigir y a exigirse

9 Russell había oído decir que Wittgenstein queria hacerse monje y retirarse en un convento.

10 Carta de 1919 citada por Anscambo en An Introduction to Wittgenstein's Tractatus, 1959.

11 A. Janik y S. Toulmin, Wittgenstein's Vienna, 1973. Este libro inspira buena parte de estas páginas aunque es bueno leer, ver, escuchar a intelectuales y artistas de Viena de este periodo. En cuanto a las místicas: todas tienden a mostrar más que a demostrar, pero la de Wittgenstein no es una mistica teísta aunque sea, como veremos, ascética a su modo. 
un rigor que sitúa en primerísimo lugar las actitudes éticas. Entre ellos podemos recordar a Karl Kraus, Arnold Schönberg, Adolf Loos, Robert Musil y, más reciente en el tiempo pero no menos crítico, autocrítico y riguroso, Elías Canetti.

Karl Kraus pensaba que "solamente una operación quirúrgica profunda podía salvar a la sociedad". Penetrante escritor de epigramas, como escritor de epigramas muy bien escritos será el Wittgenstein del Tractatus y como lo había sido antes que él uno de sus maestros, Lichtenberg, Kraus llenaba su "anti-periódico" Die Fackel, que Wittgenstein siguió recibiendo ya alejado de Viena, de invectivas, polémicas, y deseos de disciplina intelectual, vital, moral. Kraus no cree que existan ideas morales o inmorales: hay hombres que pueden ser morales, amorales o inmorales. Por decirlo con Elías Canetti, Karl Kraus "unía la esfera de la moral y de la literatura. En el caos intelectual que siguió a la Primera Guerra Mundial, no hubo tal vez nada más necesario que esta conjunción". Por esto, Karl Kraus mostró siempre un odio absoluto a la guerra. Añade Canetti acerca de este hombre admirable y por él admirado: "Desde que lo escuché no me ha sido posible no escuchar." 12

Una actitud paralela a la de Karl Kraus, en Arnold Schönberg. Nuevamente encontramos en el compositor un mismo deseo de exactitud y disciplina. ¿No pensaba Schönberg haber realizado en la música un cambio equivalente al que en su época y momento realizaron Monteverdi o, más tarde, Bach? Schönberg no creía en la inspiración. Componer era, para Schönberg, construir toda una lógica y construirla hasta tal punto que la melodía -esencia de toda música para Mozart- fuera un resultado de la forma. La disciplina que Schönberg exigía a sus discípulos era la misma que se exigía a sí mismo y esta disciplina era tanto intelectual como moral.

Contemporáneo de Schönberg. Loos, cuya arquitectura influyó en la de Wittgenstein, reaccionaba contra los ornamentos más o menos artificiosos del Art Nouveau y levaba a cabo una obra diseñada, escueta y, hay que repetirlo, lógica. En buena medida Loos fue a la arquitectura lo que la parte decible del Tractatus es al pensamiento; Adolf Loos quien una vez dijo, hablando a Wittgenstein, "ipero si yo soy tú!" 13 En efecto, todo lo decible en el Tractatus sigue un diseño perfecto, escueto, difícil y al mismo tiempo claro como son claras las obras de su amigo arquitecto. Cuando Wittgenstein construya la casa de una de sus hermanas estará influido por Loos y por este otro arquitecto, gran amigo, que fue Engelmann.

Wittgenstein vivió muchos años todo este ambiente de Viena $-\mathrm{y}$ esto

12 Elías Canetti, La conciencia de las palabras, 1981.

13 Paul Enkelmann, Letters from Wittgenstein with a Memoir, 1967. 
es lo que no siempre han percibido sus discípulos cuando tratan de descifrar o aplicar su pensamiento. ${ }^{14}$ Lo vivió y lo respiró desde su infancia, tanto en la casa de sus padres donde iban Clara Schuman, Gustav Mahler, y alguna vez Brahms, como en el castillo de Schönborn donde una de sus hermanas había alquilado un piso, también centro de reuniones intelectuales y artísticas de Viena. La filosofía de Wittgenstein refleja las preocupaciones de su ciudad natal y, en todo lo que considera decible, se expresa con la misma precisión y rigor de un Karl Kraus; muestra además, como Kraus, un especial interés, de orden vital, hacia las cuestiones éticas.

De la familia de Wittgenstein, de origen judio, sabemos que fue adinerada y culta. Su padre, nacido en Leipzig, se instaló en Viena. Sus hijas, hermanas del filósofo, navegaron por varios mares de la cultura. Ludwig, el más joven de los hermanos, nació en 1889. Hasta la edad de catorce años 'Wittgenstein estudió en su casa; estudió después en la Realschule de Linz, y en 1911 viajó a Manchester para perfeccionar sus estudios de ingeniería. En Manchester leyó a Russell, y a partir de este momento se dedicó a la filosofía. Voluntario en la Primera Guerra Mundial, terminó en las trincheras el Tractatus (1918), libro que en 1919 presentó como tesis doctoral en el Trinity College de Cambridge. Su vida inquieta y repetidamente acongojada le llevó a zigzaguear de un trabajo a otro, de una a otra ocupación. En 1920 fue jardinero en Karlsternerburg, cerca de Viena; entre 1920 y 1922 se dedicó a ser maestro de escuela primaria en Tattenbach. Su afecto hacia los niños le hizo pasar dos años de cierta felicidad; jugaba con ellos y les contaba cuentos de hadas mientras, por su parte, se aficionaba a los westerns y a las novelas policiacas. En 1921 lo encontramos, aislado y solitario, en una cabaña propia en un fiordo noruego; habrá de regresar a su soledad noruega. Cuando regresa a Austria, donde vuelve a dedicarse a la enseñanza elemental, construye, influido por su alter ego Loos y por Engelmann, la casa de una de sus hermanas (1926-1928) en Kurmangasse, trabajo en buena medida terapéutico que lo aleja de sus frecuentes angustias. Rico heredero, regala toda su fortuna, y piensa convertirse en monje. A partir de 1929 es profesor en Cambridge; da sus clases o sus pláticas, siempre muy libres en su "roer", hasta 1936, año en que regresa a Noruega. Aquí empieza a redactar ese libro no ya epigramático, sino interrogativo, que en 1945 se publicará con el título de

14 Es justo decir que algunos se han referido a la "mística" de Wittgenstein (entre ellos Anscombe y Max Black). No lo es menos recordar que, entre los seguidores de las ideas de Wittgenstein, ha predominado la influencia de la segunda época, la de las Investigaciones filosóficas que, si bien se alejan menos de lo que podría parecer del Tractatus (véase el libro de Janik y Toulmin ya citado), no dejan de plantear otra problemática y desarrollar otros métodos. 
Investigaciones filosóficas. En 1937 regresa a Cambridge, donde sucede a G. E. Moore. A partir de este año influye decisivamente en los jóvenes estudiantes ingleses. Voluntario en un hospital de Londres durante la Segunda Guerra, visita rápidamente los Estados Unidos (1947). Nuevo retraimiento y retiro, ahora en una granja islandesa. En 1951 muere, recién cumplidos los sesenta y dos años.

Tales algunos datos escuetos. ¿Por qué citarlos aquí? Porque ponen de manifiesto algo de su carácter, y porque no dejan de tener influencia en el talante de su filosofía. Algo es esencial en esta personalidad precisa, honda y dispersa: la tendencia a la ansiedad solamente corregida cuando está cerca de los niños o construyendo la casa de su hermana. Tiene razón George Pitcher: "Los demonios le persiguieron hasta el fin." 15 Y estos demonios fueron reales. Psicológicamente, este hombre angustiado estaba destinado a acercarse y apartarse al mismo tiempo de la religión y de su propio "misticismo". Por otra parte, hay en Wittgenstein algo de romántico que no se encuentra entre sus discípulos. Su vinculación con el romanticismo alemán fue profunda, tan profunda como la de Heidegger con quien, algunas veces, Wittgenstein parece tener alguna afinidad. ${ }^{16}$

En las cartas a Engelmann, Wittgenstein revela, como lo hará a veces en sus cartas a Russell, su verdadera preocupación, su verdadera angustia. Así, en 1920, escribe a su amigo: "...todavía temo que el diablo venga un día y me lleve. ¡No bromeo!" y, ese mismo año: "Todos los diablos del infierno se sueltan dentro de mi." Recordamos otra vez su motivo de gran angustia: "todo se reduce a que no tiene fe". En 1925, por otra parte, escribe a Engelmann desde Cambridge: "Estoy en Inglaterra, vivo con Keynes, pero estoy tan lejos de la claridad como siempre"... "Todavía estoy indeciso en cuestiones fundamentales." ¿Cuáles pueden ser estas cuestiones? Probablemente las que no pueden decirse pero que, a veces, pueden mostrarse.

Según Engelmann, Wittgenstein fue siempre un hombre verdaderamente preocupado por cuestiones religiosas pero éstas no solian remitir a un Dios creador sino a una de sus obsesiones: el Juicio Final.

Es importante recordar las lecturas no filosóficas de Wittgenstein;

15 Véase George Pitcher, obra citada.

16 No se trata aqui, insensatamente, de hacer la introspección - ¿cómo hacerla?de Wittgenstein. Se trata de hacer ver cómo este hombre tenía capacidad religiosa, una capacidad acaso frenada por su propia angustia. ¿Qué lo hace afín a Heidegger? Su relación intima con los poetas románticos de Alemania, cierta tendencia mística que en Heidegger se encuentra sobre todo en los escritos de los años de 40 a 60 -Logos, Aletheia, Meira, Serenidad y otros más. No quiero confundir al lector. El Angst existencial de $¿ Q u e ́$ es metafisica? poco o nada tiene que ver con la ansiedad de orden psicológico que se muestra en la vida de Wittgenstein. 
todas ellas son reveladoras. En 1917, en plena guerra, pide que le envien a las trincheras una pequeña Biblia al mismo tiempo que pide las Leyes básicas de la aritmética de Frege. Pero sus modelos parecen haber sido, ante todo, Goethe, Lichtenberg, a quien cita en sus obras, Uhland, Möricke, Rilke, von Hofmannsthal, Trakl, los pensadores semireligiosos (de una suerte de religión humanista, como Tolstoy) y Kierkegaard, a quien admiraba profundamente. Entre los místicos leyó a Eckhart y Tauler y sobre todo a este maravilloso poeta que fue Angelus Silesius. Poeta del silencio, Angelus Silesius se aproxima a los silencios callados y entredichos de la gran mística cuando escribe:

Ya basta ahora, amigo, si quieres

leer más ve y conviértete

en el Escrito y su sentido.

Hasta aquí algunos datos que podrían parecer extrafilosóficos. No lo son. $\mathrm{Y}$ si no lo son es porque un filósofo no es - no debe ser- un ente abstracto, sino una persona viva que integra en sí, y por lo tanto en su filosofía, cuanto lee, siente, vive. La biografía es parte del pensamiento, sobre todo cuando se trata de cuestiones fundamentales, es decir, de cuestiones indecibles. ${ }^{17}$

Pero ¿qué significa en Wittgenstein "lo místico"?; ¿cuál es la experiencia que está en el límite o más allá de los límites?

A lo largo del Tractatus insiste, persiste la presencia del límite. Volvamos al "Prólogo" del libro. Dice Wittgenstein muy claramente que su obra "quiere trazar unos límites al pensamiento, o mejor, a la expresión de los pensamientos; porque para trazar un límite al pensamiento tendríamos que ser capaces de pensar ambos lados de este límite y, por consiguiente, tendríamos que ser capaces de pensar lo que no se puede pensar". Una primera lectura de estas frases podría hacer creer que no podemos decir nada acerca de lo que está más allá de los límites, lo cual es cierto. No es menos cierto que precisamente aquello que no se puede pensar se puede mostrar y que así, en cierta forma, vemos el otro lado del límite. En otras palabras: lo indecible no es del todo indecible, porque podemos ponerlo de manifiesto de manera alusiva, aunque no lógica.

En otras palabras Wittgenstein quiere, como Kant, poner claros lími-

17 Por esto son aquí esenciales los Notebooks y no sólo el Tractatus. 
tes al pensamiento humano; a diferencia de Kant, Wittgenstein, dubitativo o aun escéptico, no podrá edificar un sistema de moral.

El Tractatus concluye con las siguientes frases:

"Mis proposiciones son esclarecedoras de este modo: que quien me comprende acaba por reconocer que carecen de sentido, siempre que el que comprenda haya salido, a través de ellas, fuera de ellas. (Debe, pues, por así decirlo, tirar la escalera después de haber subido por ella.) Debe superar estas proposiciones; entonces tiene la justa visión del mundo." ${ }^{18}$

De pronto todo se ilumina. Si por una parte lo decible aclara algunas cuestiones importantes, por otra, y fundamentalmente, es la vía ascética que puede conducir a lo "místico". Por esto, cuando se alcanza lo que se muestra, las proposiciones previas son "sin sentido" y, sobre todo, por esto, todo el aparato sistemático del Tractatus es lo que aquí debe nombrarse vía ascética. Así, Ayer se equivocaba totalmente cuando sostenía que Wittgenstein estaba diciendo que quienes hablaban de lo místico no habían dicho sino tonterías. Demasiado influido estaba Ayer por el positivismo lógico para poder entender a Wittgenstein. Tampoco me parece convincente la postura de Anscombe, quien dedica muy breves páginas a la cuestión de la mística, más para descartarla que para entenderla claramente. Seguiré mi propia hipótesis: Wittgenstein explica el mundo para superar su explicación y, acerca de esta superación, tiene mucho que mostrarnos aunque no que decirnos ni demostrarnos. En este sentido es totalmente pertinente uno de los epigramas del Tractatus: "Nosotros sentimos que incluso si todas las posibles cuestiones científicas pudieran responderse, el problema de nuestra vida no habría sido más profundamente penetrado." $(T \text { 6.52. })^{19}$

Las proposiciones que tienen sentido cuando se refieren a los hechos del mundo "no pueden expresar nada más alto" ( $T$ 6.42). "Mostrar" lo más alto consiste en sugerir lo que está en los límites o más allá de ellos. ¿En qué sentido podemos mostrar lo que remite a la ética y la estética una vez que sabemos que "es claro que la ética no puede expresarse", que la "ética es trascendental", que "ética y estética son lo mismo"? (T 6.421).

La ética no puede expresarse porque es trascendental y, por lo tanto, está más allá de los límites del lenguaje. Por otra parte, la identificación entre ética y estética ha sido interpretada alguna vez -así por Engelman - en este sentido: ética y estética no son lo mismo, pero ambas son

18 Véase, Max Black, A Companion to Wittgenstein's Tractatus, 1964. Según Max Black la imagen de la escalera procede de Mauthner y, lejanamente, de Sexto Empírico.

19 A partir de aquí las referencias al Tractatus serán señaladas por la letra $T y$, además, por el número correspondiente al epigrama. 
indecibles. Algo hay de verdad en esta interpretación, pero si leemos cuidadosamente a Wittgenstein veremos que, más de una vez, identifica ética y estética. En efecto, en los Notebooks Wittgenstein muestra algo acerca de la ética y la estética. Al referirse a la felicidad —obsesión constante-Wittgenstein parece separar arte y vida —estética y ética-cuando dice: "La vida es seria, el arte es alegre." Pero esta frase no puede leerse de manera aislada. Si aceptamos que "el fin del arte es lo bello", podemos afirmar que ". . .lo bello es lo que hace feliz". Así, la felicidad moral y la belleza quedan entrelazadas.

Muy poco nos dice Wittgenstein, en el Tractatus, sobre ética y estética. Por lo que se refiere a la ética, los comentarios, referencias, apostillas, son frecuentes en los Notebooks.

Sabemos, lo cual es poco saber, que "el mundo de los felices es distinto al mundo de los infelices" ( $T$ 6.423). Pero sabemos también, gracias a los Notebooks, que la "vida buena" debe ser vista sub specie aeternitatis. En otras palabras - y aquí ya estamos de lleno en el reino de das Mystische_, que esta manera de ver da sentido a la vida y puede permitir que los hombres alcancen la felicidad: "para vivir felizmente debo estar de acuerdo con el mundo. $Y$ esto es lo que 'ser feliz' significa". Ignoramos si puede hablarse de una conciencia moral, pero no ignoramos "que me hace infeliz haber ofendido a tal o cual persona". Y en esta parte de los Notebooks termina Wittgenstein diciendo, exclamativamente: " $\mid V i v e$ felizmente!... Quien viva feliz no debe tener miedo. No debe tenerlo ni aun ante la muerte." ¿Cómo alcanzar la felicidad? Por las vías y rutas del conocimiento y "a pesar de las miserias de este mundo".

En la idea de la felicidad que Wittgenstein muestra aparece cierta tendencia al desasimiento. La felicidad entraña amenidades (o distracciones) del mundo. Para renunciar a ellas debemos vivir en el presente: "solamente un hombre que viva no en el tiempo, sino en el presente, es feliz". En efecto, vivir en el tiempo es vivir hacia la muerte, pero el que viva en el presente no vivirá la muerte que, por lo demás, "no es un acontecimiento de la vida".

La felicidad, más allá de los límites del lenguaje y de la lógica, depende en buena medida de la voluntad, es decir, de aquello que trae consigo el bien y el mal. Pero la voluntad no puede cambiar el mundo; lo que mediante la voluntad puedo alcanzar es mi propia independencia del mundo.

Mucho se ha escrito sobre la influencia de Schopenhauer en Wittgenstein. Nada más cierto. Con todo, hay que entender el sentido de esta influencia. Solemos pensar en Schopenhauer como el filósofo de la voluntad y aun de la voluntad de poder, pero si Schopenhauer es un filó- 
sofo de la voluntad, lo es en la misma medida en que ve que la voluntad es, en última instancia, negadora de nuestras voluntades. Cercano al budismo, Schopenhauer acaba por ser escéptico, pesimista y, solipsísticamente, por estar alejado del mundo. Acaso cercano a esta interpretación de Schopenhauer, Wittgenstein cree y siente que la voluntad está fuera del mundo (fuera del lenguaje y más allá del lenguaje) y escribe: "El acto de la voluntad no es un acto de la experiencia." ¿Será éste el modo y la manera de alejarnos del mundo y liberarnos de sus miserias? Probablemente.

La ética, indeciblemente, es una cuestión de vida pero "el mundo y la vida son una misma cosa" ( $T$ 5.621), y la ética no es cosa de este mundo; "debe ser una condición del mundo como la lógica", es decir, y renovadamente, reiteradamente, se trata de una condición del mundo que está más allá de las lindes del mundo. La ética de Wittgenstein -mejor, su esbozo de ética- recuerda poco a Kant, a pesar de lo que digan algunos exégetas; recuerda, más bien, a los estoicos, si por estoicismo entendemos una manera de aceptar la vida sin posibilidad de cambiar el mundo. Estamos en el corazón de lo indecible. Poco se puede hablar de ética y menos de una teoría ética en el pensamiento de Wittgenstein. Como él mismo escribe en Philosophical Grammar: "no hay proposiciones éticas; hay actos".

Wittgenstein, hombre que carece de fe -o que busca una fe perdidanos adentra más en los territorios de lo místico cuando nos muestra algo acerca de la divinidad, acerca de Dios.

Lo divino - creido o descreido - se manifiesta, según la frase de los Notebooks, como "un impulso hacia la mística". Varias veces Wittgenstein se refiere a Dios en este libro de notas. Así escribe:

Creer en Dios significa entender la cuestión acerca del sentido de la vida.

Creer en Dios significa ver que los hechos del mundo no lo explican todo.

Creer en Dios significa ver que la vida tiene un sentido.

Hasta aquí Wittgenstein parece hacer profesión de fe, aunque bien pudiera ser que el sentido de estas palabras se aplicara a quienes creen en Dios y no necesariamente a Wittgenstein. Lo más probable es que no haya en Wittgenstein una fe clara - no la hay, por lo menos, de manera explícita-; hay que pensar, con Norman Malcolm, que hay en él una "posibilidad de fe".

Dios da sentido a la vida. Pero ¿cuál es el sentido de la palabra "Dios" en el contexto de la obra de Wittgenstein? 
En algunos casos Dios es visto como el Ser del cual dependemos y, así, la palabra "Dios" es sinónima de la palabra "Destino"; en otros casos la divinidad se escinde y adquiere un doble significado más allá de los límites: el mundo, considerado en su totalidad limitada, y el "Yo" dependiente (un "yo" que no es personal, sino trascendental).

Estos varios y diversos sentidos de la palabra "Dios" no dejan de ser ambiguos. Trataré de precisarlos. Antes es bueno recordar que la experiencia mística - centrada en la divinidad- es religiosa, sea cual sea la fe o falta de fe de Wittgenstein. Pocos han visto el sentido de esta mística como Ramsey, quien escribía en Foundations: "El sentimiento místico es el sentimiento de que el mundo no lo es todo, que existe algo fuera de él, su 'sentido' o su 'significado'." 20

Zemach intentó buscar los distintos sentidos de la palabra "Dios" en el Tractatus y los Notebooks. Por una parte, Dios sería "la totalidad de los hechos"; por otra sería el "Destino", el "Mundo" — lo cual acercaría a Wittgenstein a un vago panteísmo? Pero, además, la palabra "Mundo" es sinónimo de la palabra "Vida" (y por lo tanto de "Dios") "porque la vida es la totalidad de los hechos" ( $T$. 5.621) y - ¿nueva tentación panteísta? - Dios es como "se presentan las cosas". Zemach trata de ordenar esta serie de resbaladizas equivalencias -o pseudoequivalencias- y escribe: "Empezamos siguiendo algunas de las proposiciones de los Notebooks por una simple identificación con el mundo... pero esta fórmula no es exacta, ya que Wittgenstein concibe a Dios como un trascendental". Lo cual significa que Dios no es el mundo y, por lo tanto, que no existe tentación panteísta en Wittgenstein. Prosigue Zemach: Dios no se revela en el mundo. Si seguimos analizando otras proposiciones que se refieren a Dios y a lo místico, encontramos que el no-ser revelado en el mundo puede significar un manifestarse como límite del mundo... Ser el límite de un territorio significa ser la forma general, es decir, la posibilidad de este territorio; ser una forma es ser su sentido. Puesto que forma y esencia son idénticas, Dios es el sentido y la esencia del universo, la esencia del mundo, es decir, la totalidad de los hechos, y es la forma general de las proposiciones y la forma general de las proposiciones es idéntica al concepto de Dios. ${ }^{21}$

20 Citado por Max Black en la obra ya mencionada.

21 Véase el texto de Zemach en Essays on Wittgenstein's Tractatus, selección de I. M. Copi y R. W. Beard, 1966. Dos acotaciones: cuando en la frase citada Ramsey habla del "mundo" no utiliza este término en el sentido de Wittgenstein sino en el sentido común y corriente: el mundo, en efecto, esta nuestra tierra. Wittgenstein oscila entre la afirmación de que Dios es el mundo y la afirmación según la cual -más allá de límites- Dios es la condición del mundo de la misma manera que la lógica es condición de un tipo de pensamiento y cálculo. En cuanto a la voluntad, aun cuando pensemos que llegue al mundo y sea una forma de nombrar a Dios, no deja de ser voluntad, en el sentido schopenhaueriano que indiqué más arriba. 
La interpretación de Zemach no es incorrecta; habría que completarla. De hecho, Wittgenstein no parece identificar lo místico como el límite del mundo sino como aquello que está más allá de este límite (parábola de la escalera que debemos tirar después de haber subido por ella). Dios podría ser visto como la condición de esta totalidad finita que es el mundo. A esta serie de atributos — verdaderamente ambiguoshabria que añadir el universo de los Valores y la Voluntad -otro de los trascendentales en Wittgenstein, aunque esta Voluntad fuera la negación de la voluntad. La voluntad ingresa al mundo a través de nuestra voluntad y nos aleja del mundo mediante nuestra voluntaria desvoluntad. Además, el bien y el mal no son cosas de esta tierra; llegan al mundo por la voluntad.

El dios de Wittgenstein -el que le preocupa que crea o no en Él_ está presente en una suerte de letanía de los Notebooks:

¿Qué sé yo acerca de Dios y del sentido de la vida? Sé que el mundo existe.

Que estoy situado en él como mi ojo en el campo visual.

Que algo acerca de él es problemático, a lo cual llamamos significado.

Que este significado no está en (el mundo) sino fuera de él.

Que la vida es el mundo.

Que mi voluntad penetra en el mundo.

Que mi voluntad es buena o es mala.

Así, que el bien y el mal están de alguna manera relacionados con el mundo.

El sentido de la vida, es decir, el sentido del mundo, podemos llamarlo Dios.

Y relacionar con esto la comparación de Dios con un Padre.

Orar es pensar acerca del sentido de la vida.

No puedo doblegar los acontecimientos del mundo a mi voluntad; soy totalmente impotente.

Solamente puedo hacerme independiente del mundo $-\mathrm{y}$ así, en cierto sentido, dominarlo- renunciando a cualquier influencia sobre los acontecimientos.

Dos cosas parecen claras: por una parte Dios está fuera del mundo y, schopenhauerianamente, mi voluntad es noluntad.

Por otra parte, el lenguaje empleado por Wittgenstein en lo que he llamado letanía y que él prácticamente califica de oración es varias veces probabilístico ("problemático", "de alguna manera", "en cierto sentido"); es probabilístico y un si es no es vago. Se trata del lenguaje de la indecisión. Tenía razón Malcolm: en Wittgenstein existe una "posibilidad" de religión. Como Kierkegaard, Wittgenstein quiso realizar 
un saltum mortale; no parece que lo haya conseguido como lo consiguió -a través de amor y fe, a través de "obra de amor"- Sören Kierkegaard.

El "impulso" de Wittgenstein recuerda a algunos prerrománticos o románticos. También ellos tuvieron la experiencia del límite y la trascendencia del límite. Así Lichtenberg, a quien había leído repetidas veces Wittgenstein: " $\mathrm{i} \mathrm{Si}$ cuando menos hubiese traspasado el punto límite!, 'qué deseo hay en mi, Dios mío, de ese instante en que el tiempo ya no será para mí el tiempo...l" La angustia existencial, el temor a la nada, la continua referencia al Juicio Final, las menciones de Dostoievski y sus citas de Dmitri Karamazov sitúan a Wittgenstein lejos de la claridad, y lo colocan no ya en el ambiente de lucidez de un Kraus o un Loos sino en la tradición del romanticismo alemán y aun del nihilismo, hijo y heredero del romanticismo. Es necesario decir que el Wittgenstein clásico y preciso del Tractatus quiere ir más allá de su clasicismo para acercarse a lo que le urge resolver y no puede resolver: acercarse a lo místico, tan necesario para él como vago y motivo de dudas existenciales. No bauticemos a Wittgenstein de existencialista; recordemos que su vocación cardinal fue la del hombre religioso muchas veces sumergido en la angustia y la zozobra. Y cuando más positivamente ve Wittgenstein la experiencia del límite y del más allá del límite es cuando ve el mundo como un "milagro", el milagro "de que el mundo exista" - tales son sus palabras - y que sea imposible "imaginarlo como no existente" - tales también sus palabras.

Alguna vez, al referirse a la experiencia religiosa, Wittgenstein dijo, con anhelo pero acaso carente de la experiencia de la salvación, que deseaba "sentirse absolutamente a salvo". En cuanto a su experiencia intima, nada podremos saber de ella; acaso se haya acercado a la de Eduard Möricke — su poeta admirado_ cuando éste decía:

Mundo, déjame en paz, no me tientes con regalos de amor, deja que mi corazón guarde su gozo y su dolor para sí mismo. 